\section{Use of locked nucleic acid oligonucleotides as hybridization/FRET probes for quantification of $16 S$ rDNA by real-time PCR}

\author{
Oliver Goldenberg ${ }^{1}$, Olfert Landt ${ }^{2}$, Ralf R. Schumann ${ }^{1}$, UIf B. Göbel ${ }^{1}$, \\ and Lutz Hamann ${ }^{1}$ \\ ${ }^{1}$ Charité University Medical Center and ${ }^{2}$ TIB MOLBIOL, Berlin, Germany
}

BioTechniques 38:29-32 (January 2005)
Quantification of bacterial species from the highly diverse human gastrointestinal microflora $(1,2)$ is a great technical challenge (3). Since several diseases are associated with an altered composition of the gastrointestinal microflora $(2,4,5)$, methods for quantification of specific bacterial species or genera are of great importance to monitor the population dynamics within this microbial ecosystem. Various methods for analyzing the composition of complex microbiota have been developed (reviewed in Reference 6). As most intestinal bacterial species are yet uncultured, quantification by real-time PCR seems to be the method of choice. Real-time PCR allows quantification using SYBR ${ }^{\circledR}$ Green or probe-based systems, [e.g., TaqMan ${ }^{\circledR}$ or hybridization/fluorescent resonance emission transfer (FRET) probes] (7). The major advantage of probes is the sequence-specific detection, whereas SYBR Green detection often results in a trade-off between sensitivity and specificity due to detection of unspecific amplification products.

Unfortunately, species- or genusspecific sequences of the 16S rRNA genes required for specific detection of bacteria are very short, thus preventing the use of DNA-based probes. Conventional DNA probes of approximately $20 \mathrm{bp}$ in length are necessary to reach Thus, the quantification of speciesspecific bacterial DNA with probes requires species-specific amplification using two primer pairs for comparing two different bacterial species. Quantitative comparison of two PCR amplifications demands tremendous a suitable melting temperature $\left(\mathrm{T}_{\mathrm{m}}\right)$. "fine-tuning" due to different PCR efficiencies resulting from varying binding kinetics of the two different primer pairs. Therefore, our aim was to establish an assay to quantify different bacterial species with probes from one 16S rDNA product, generated by one pair of eubacterial-conserved primers. This product spans different species-specific regions, allowing the quantification by individual specific probes. Such an approach using minor groove binding (MGB) TaqMan probes has recently been published (8). The addition of the minor groove binding peptide to the TaqMan probes resulted in an increased $T_{m}$, thereby allowing for the design of very short species-specific probes. Alternatively, the incorporation of locked nucleic acid (LNA) oligonucleotides within the probes also results in an increased $\mathrm{T}_{\mathrm{m}}$ due to their rigid conformational structure (9). Several applications for LNA technology have been described within the last 2 years, including

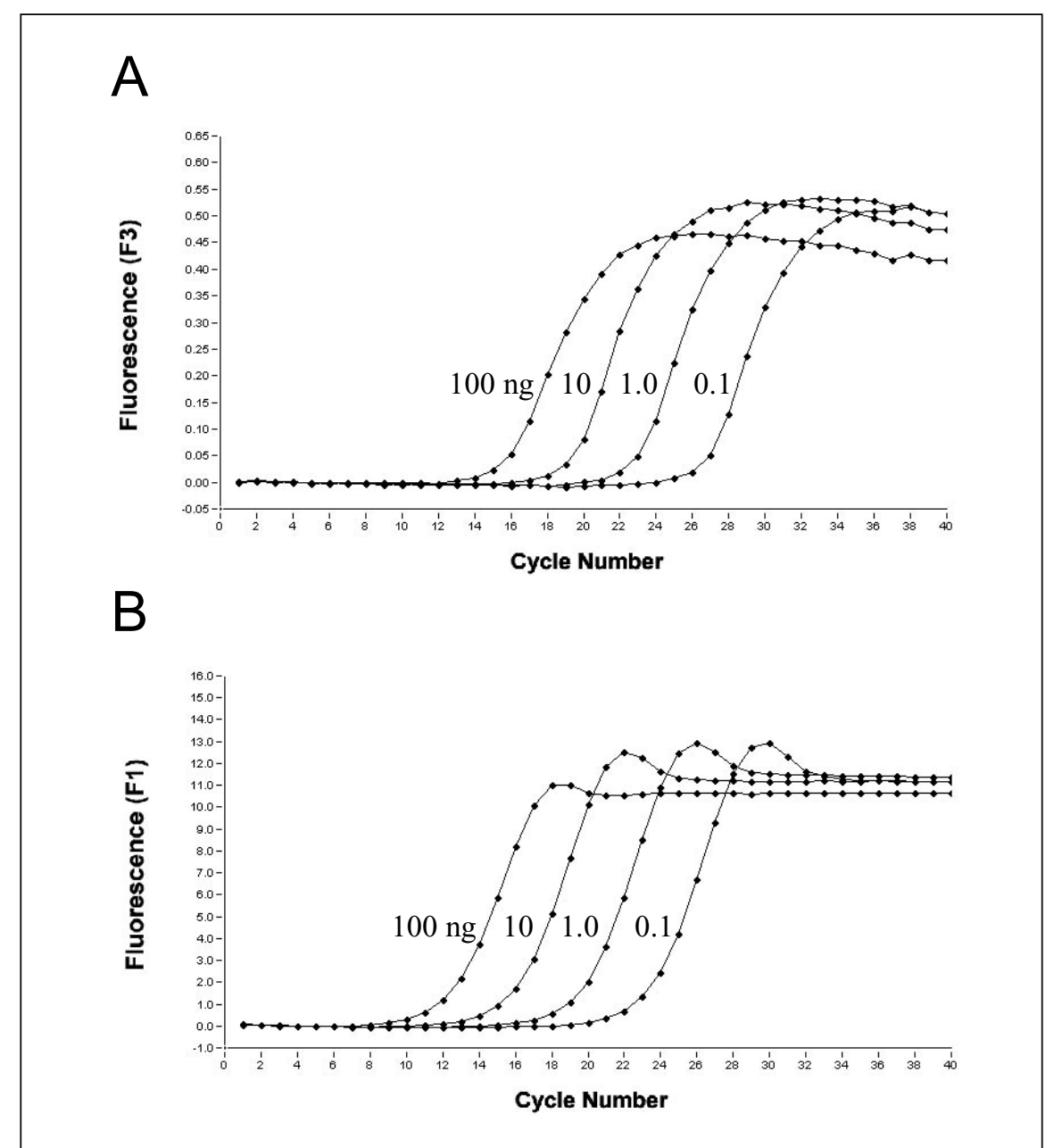

Figure 1. Comparative detection of fluorescent signal development between SYBR Green and locked nucleic acid (LNA) hybridization/fluorescent resonance emission transfer (FRET) probes using DNA from human feces. (A) Hybridization/FRET probes. (B) SYBR Green. 
single nucleotide polymorphism (SNP) analysis, 5'-nuclease assay, expression profiling, and fluorescence in situ hybridization (FISH) analysis (10-15).

We demonstrate here that LNAcontaining oligonucleotides can also be used as hybridization/FRET probes for detection and quantification of short species-specific 16S rDNA sequences by real-time PCR. Based on this novel application, we established a sensitive assay for rapid quantification of bacterial 16S rDNA using hybridization/FRET probes containing LNA nucleotides.

The assay is based on the amplification of a 354-bp fragment of the $16 \mathrm{~S}$ rRNA target gene from eubacterial DNA. DNA was isolated by a conventional phenol/chloroform extraction from human stool samples representating the complex gastrointestinal microbial community. Eubacterial-conserved primers for amplification used were as follows: sense, 5'-CATGGRTGTCGTCAGCTCGTG$3^{\prime}$ and reverse, 5'-TGACGGGCGGTGTGTACA-3' ( $\mathbf{R}=$ A or $\mathrm{G}$, due to variations at this position). The product was detected with eubacterial-conserved hybridization/FRET probes (EuBdonor and EuBacceptor) containing LNA nucleotides: EuBdonor, 5'-ACGTCATCCCC-3' labeled with fluorescein at the $3^{\prime}$ end and EuBacceptor, 5'-CCTTCCTC$3^{\prime}$ labeled with LC-705 at the $5^{\prime}$ end. The LNA nucleotides are shown in bold. The eubacterial-conserved regions within the 16S rRNA genes for designing primers and probes were chosen according to the analysis of domain-specific $16 \mathrm{~S}$ primers by Baker and Cowan (sense, pos. 1054-1074; reverse, pos. 1391-1408; EuBdonor, pos. 1184-1194; EuBacceptor, pos. 1174-1182) (16). The insertion of the respective LNA nucleotides resulted in $\mathrm{a} \mathrm{T}_{\mathrm{m}}$ shift from $33^{\circ}$ to $63^{\circ} \mathrm{C}$ (EuBdonor) and below $26^{\circ}$ to $65^{\circ} \mathrm{C}$ (EuBacceptor) compared to DNA oligonucleotides without LNAs. PCR was performed in a total volume of $20 \mu \mathrm{L}$ containing 4 $\mu L$ FastStart DNA Master ${ }^{\text {PLUS }}$ hybridization/FRET (Roche Diagnostics GmbH, Mannheim, Germany), $5 \mu \mathrm{L}$ DNA, $0.25 \mu \mathrm{M}$ primers, and $0.2 \mu \mathrm{M}$ probes. Amplification in the LightCycler ${ }^{\circledR}$ instrument (Roche Diagnostics
$\mathrm{GmbH}$ ) was according to the following parameters: initial denaturation at $95^{\circ} \mathrm{C}$ for $10 \mathrm{~min}, 40$ cycles of denaturation at $95^{\circ} \mathrm{C}$ for $0 \mathrm{~s}$, annealing at $60^{\circ} \mathrm{C}$ for $10 \mathrm{~s}$, and extension at $72^{\circ} \mathrm{C}$ for $15 \mathrm{~s}$. Fluorescence intensity was determined during each cycle at the end of the annealing step. By performing 10-fold dilution steps of $100 \mathrm{ng}$ target DNA, it could be seen that LNA-containing hybridization/FRET probes are well suited for specific product detection (Figure 1A).

To compare this new detection method with a conventional detection method, we performed a similar PCR run with the same primers and template DNA using SYBR Green for detection (Figure 1B). A comparison of both assays showed similar results regarding fluorescence generation, demonstrating that LNA-containing oligonucleotides can be applied as hybridization/FRET probes for real-time PCR (Figure 1). However, cycle threshold $\left(\mathrm{C}_{\mathrm{t}}\right)$ values were found to be approximately 3.2 cycles lower with the SYBR Green assay, indicating a higher sensitivity of SYBR Green compared to hybridization/FRET probes, most likely due to the fact that a greater number of SYBR Green molecules are incorporated by the product.

To show species-specific detection, we designed LNA/FRET probes specific for the Bacteroides genus as follows: 5'-LC-640-AGCCGACACCT$3^{\prime}$ and 5'-CGTTATGGCACTT-FL-3'. Amplification with the same conditions as described above, and Bacteroidesspecific detection is shown in Figure 2. Using 10-fold dilutions of Bacteroides thetaiotaomicron DNA yields strong

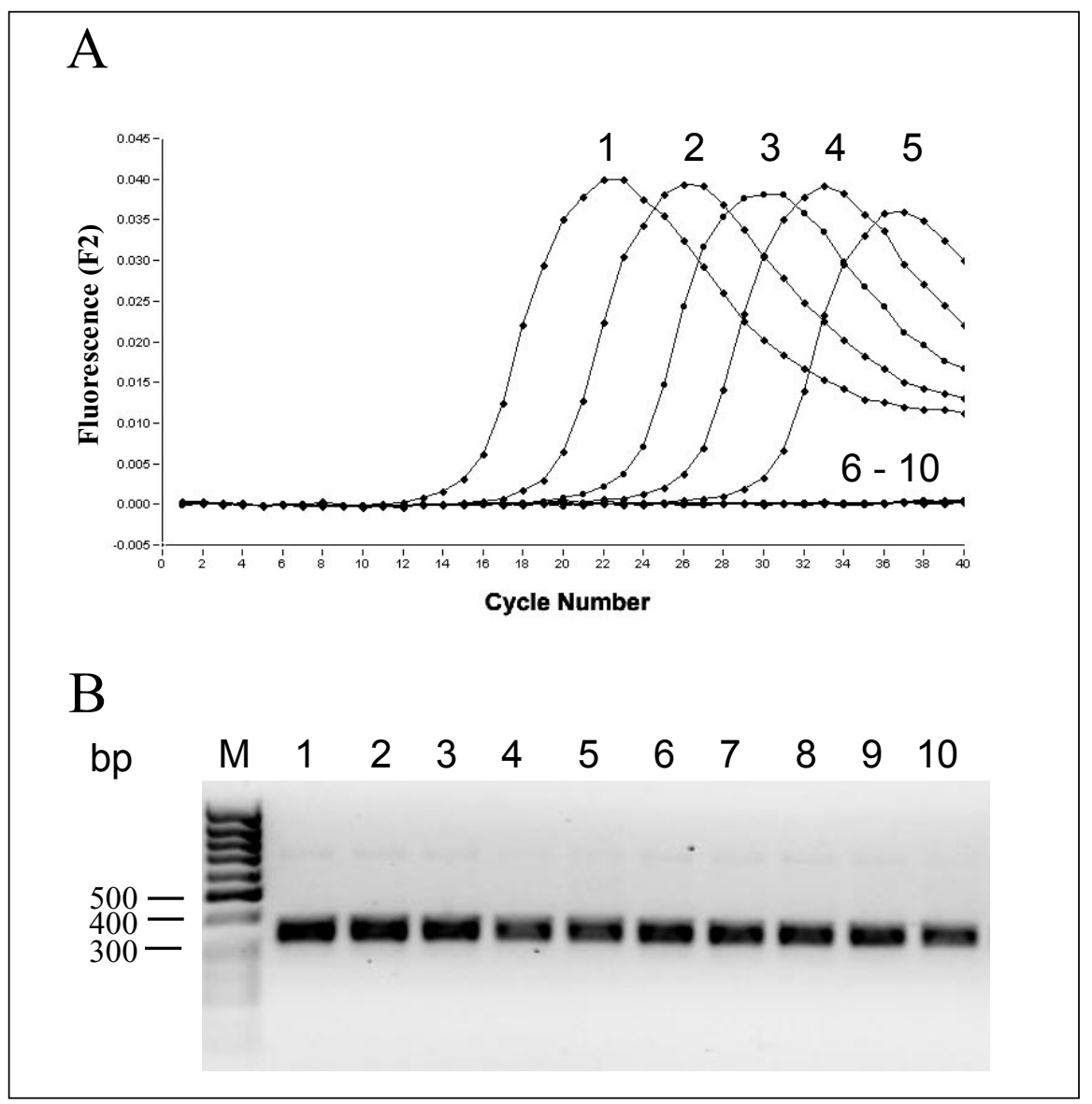

Figure 2. Sensitivity and specificity of locked nucleic acid (LNA) hybridization/fluorescent resonance emission transfer (FRET) probes specific for the Bacteroides genus. (A) Fluorescent signal generation using $100 \mathrm{ng}$ (lane1), $10 \mathrm{ng}$ (lane 2), $1 \mathrm{ng}$ (lane 3), $0.1 \mathrm{ng}$ (lane 4), and $0.01 \mathrm{ng}$ (lane 5) of Bacteroides thetaiotaomicron DNA. To control specificity $100 \mathrm{ng}$ DNA of Klebsiella planticola (lane 6), Enterococcus faecalis (lane 7), Bacillus cereus (lane 8), Clostridium sporogenes (lane 9), and Bifidobacterium longum (lane 10) were used. (B) Successful amplification was shown by agarose gel electrophoresis. 
signal generation (Figure 2A, lanes $1-5)$. In contrast, $100 \mathrm{ng}$ of five control DNAs from Klebsiella planticola, Enterococcus faecalis, Bacillus cereus, Clostridium sporogenes, and Bifidobacterium longum, each containing three mismatches per probe, gave no fluorescence signal even after 40 cycles of amplification, indicating high specificity of the probes (Figure 2A, lanes 6-10). Successful amplification of all DNAs was confirmed by agarose gel electrophoresis (Figure 2B).

Taken together, the results demonstrate that LNA-based hybridization/ FRET probes are suitable alternatives to the recently published MGB probes in quantitative real-time PCR of $16 \mathrm{~S}$ rDNA and extend the potential of using short detection probes with respect to dye chemistry and PCR instruments. Employing LNA oligonucleotides may permit the use of significant shorter probes, as LNA-containing hybridization/FRET probes of $8-11 \mathrm{bp}$ are working as well as MGB probes of 13-18 bp (8). Furthermore, both assays should be able to discriminate between species that differ in only 1 or 2 bp due to the significant decrease in the $T_{m}$ by either mismatched LNA-containing probes or MGB probes.

\section{ACKNOWLEDGMENTS}

This work was supported by the Bundesministerium für Bildung und Forschung (BMBF, CAPNetz, Project C5) and the Deutsche Forschungsgemeinschaft (DFG, project GO 363/10-1).

\section{COMPETING INTERESTS STATEMENT}

The authors declare no competing interests.

\section{REFERENCES}

1.Boriello, S.P. 1986. Microbial flora of the gastrointestinal tract, p. 2-16. In M.J. Hill (Ed.), Microbial Metabolism in the Digestive Tract. CRC Press, Boca Raton.

2.Kalliomaki, M. and E. Isolauri. 2003. Role of intestinal flora in the development of allergy. Curr. Opin. Allergy Clin. Immunol. 3:15-20.

3.von Wintzingerode, F., U.B. Gobel, and E. Stackebrandt. 1997. Determination of microbial diversity in environmental samples: pitfalls of PCR-based rRNA analysis. FEMS Microbiol. Rev. 21:213-229.

4.Guarner, F. and J.R. Malagelada. 2003. Gut flora in health and disease. Lancet 361:512519.

5.Seksik, P., L. Rigottier-Gois, G. Gramet, M. Sutren, P. Pochart, P. Marteau, R. Jian, and J. Dore. 2003. Alterations of the dominant faecal bacterial groups in patients with Crohn's disease of the colon. Gut 52:237242.

6.Zoetendal, E.G., C.T. Collier, S. Koike, R.I. Mackie, and H.R. Gaskins. 2004. Molecular ecological analysis of the gastrointestinal microbiota: a review. J. Nutr. 134:465-472.

7.Wittwer, C.T., K.M. Ririe, R.V. Andrew, D.A. David, R.A. Gundry, and U.J. Balis. 1997. The LightCycler: a microvolume multisample fluorimeter with rapid temperature control. BioTechniques 22:176-181.

8.Ott, S.J., M. Musfeldt, U. Ullmann, J. Hampe, and S. Schreiber. 2004. Quantification of intestinal bacterial populations by real-time PCR with a universal primer set and minor groove binder probes: a global approach to the enteric flora. J. Clin. Microbiol. 42:2566-2572.

9.Koshkin, A.A., K.S. Sanajay, P. Nieslen, K.V. Rajwanshi, R. Kumar, M. Meldgaard, C.E. Olsen, and J. Wengel. 1998. LNA (locked nucleic acid): synthesis of the adenin, cytosin, guanine, 5-methylcytosin, thymidin and uracil bicyclonucleosid monomers, oligomerisation, and unprecedented nucleic acid recognition. Tetrahedron 54:3607-3630.

10.Johnson, M.P., L.M. Haupt, and L.R. Griffiths. 2004. Locked nucleic acid (LNA) single nucleotide polymorphism (SNP) genotype analysis and validation using real-time PCR. Nucleic Acids Res. 32:e55.
11.Letertre, C., S. Perelle, F. Dilasser, K. Arar, and P. Fach. 2003. Evaluation of the performance of LNA and MGB probes in 5'-nuclease PCR assays. Mol. Cell Probes 17:307311.

12.Silahtaroglu, A.N., N. Tommerup, and H. Vissing. 2003. FISHing with locked nucleic acids (LNA): evaluation of different LNA/ DNA mixmers. Mol. Cell Probes 17:165-169.

13.Simeonov, A. and T.T. Nikiforov. 2002. Single nucleotide polymorphism genotyping using short, fluorescently labeled locked nucleic acid (LNA) probes and fluorescence polarization detection. Nucleic Acids Res. 30:e91.

14.Tolstrup, N., P.S. Nielsen, J.G. Kolberg, A.M. Frankel, H. Vissing, and S. Kauppinen. 2003. OligoDesign: optimal design of LNA (locked nucleic acid) oligonucleotide capture probes for gene expression profiling. Nucleic Acids Res. 31:3758-3762.

15.Ugozzoli, L.A., D. Latorra, R. Pucket, K. Arar, and K. Hamby. 2004. Real-time genotyping with oligonucleotide probes containing locked nucleic acids. Anal. Biochem. 324:143-152.

16.Baker, G.C., J.J. Smith, and D.A. Cowan. 2003. Review and re-analysis of domainspecific 16S primers. J. Microbiol. Methods 55:541-555.

Received 21 June 2004; accepted 20 August 2004.

Address correspondence to Lutz, Hamann, Institute for Microbiology and Hygiene, Charité, Humboldt University Berlin, Dorotheenstrasse 96, 10117 Berlin, Germany. e-mail:lutz.hamann@charite.de 